\title{
Differences in stroke outcome based on sex
}

N. Shobha, DM

P.N. Sylaja, MD

M.K. Kapral, MD, FRCPC

J. Fang, PhD

M.D. Hill, MD, MSc, FCPC

For the Investigators of the Registry of the Canadian Stroke Network

Address correspondence and reprint requests to Dr. Michael D. Hill, Calgary Stroke Program, Department of Clinical Neurosciences, University of Calgary, Foothills Hospital, Rm $1242 \mathrm{~A}, 140329^{\text {th }}$ Street NW, Calgary, Alberta, T2N 2T9,

Canada

michael.hill@albertahealthservices.ca

\section{ABSTRACT}

Objective: Stroke thrombolysis may have a differential effect by sex. We sought to examine the relationship between sex and outcome after thrombolysis.

Methods: This is a retrospective cohort study of stroke patients from the Registry of Canadian Stroke Network phase 1 (June 2001-February 2002) and phase 2 (June 2002-December 2002). Variables including demographics, history, clinical data, process measures, and outcome were analyzed. The primary outcomes were the Stroke Impact Scale-16 score (SIS-16) and mortality at 6 months. We compared the outcomes of the thrombolyzed and nonthrombolyzed cohorts and examined the data for a tissue plasminogen activator (tPA)-by-sex interaction on the 2 primary outcomes.

Results: The overall proportion of patients who achieved an excellent outcome (SIS-16 > 75) was not different by gender. However, the proportion of patients achieving an excellent outcome in the non-tPA cohort was much greater in males, with an absolute risk difference of $11.8 \%$. A multiplicative treatment by sex interaction was evident $(p=0.054)$. This interaction was not present for stroke case fatality.

Conclusions: Women fared poorly compared to men in the placebo groups, but this negative prognostic sex effect was neutralized by thrombolysis. Neurology ${ }^{\circledR}$ 2010;74:767-771

\section{GLOSSARY}

mRS = modified Rankin Score; RCSN = Registry of the Canadian Stroke Network; SIS-16 = Stroke Impact Scale-16 score; tPA = tissue plasminogen activator

Women manifest stroke differently and have worse outcomes after ischemic stroke compared to men. ${ }^{1,2}$ There is evidence that stroke thrombolysis with tissue plasminogen activator (tPA) has a differential effect by sex. While both sexes benefit, women show a greater treatment effect compared to men. This has been suggested in post hoc analyses of 6 pooled IV tPA treatment trials and in 1 intraarterial recombinant prourokinase treatment trial. ${ }^{3,4}$ The consistency of effect suggests a true association but the biologic reasons why this may occur remain obscure. Suggested mechanisms include increased early arterial patency ${ }^{5}$ but there are no convincing data to support this hypothesis.

One possible concern is that patients in the randomized trials are somehow different from those in routine practice and that such an interaction may not be present in clinical routine. We sought to examine the relationship between sex and outcome after stroke thrombolysis in a cohort of routinely treated patients from the Registry of the Canadian Stroke Network (RCSN). Our aim was to provide further evidence for or against the consistency of effect.

METHODS The methodology of the RCSN has been described in detail elsewhere. ${ }^{6}$ In phase 1 (June 2001-February 2002) and phase 2 (June 2002-December 2002), data were collected on consecutive patients with acute stroke or TIA seen at 21 (phase 1) and 25 (phase 2)

From the Departments of Clinical Neurosciences (N.S., M.D.H.) and Medicine and Community Health Sciences (M.D.H.), University of Calgary, Alberta, Canada; Ananathapuri Hospital and Research Institute (P.N.S.), Thiruvananthapurum City, Karela, India; Departments of Medicine and Health Policy, Management and Evaluation (M.K.K.), Faculty of Medicine, University of Toronto; Institute for Clinical Evaluative Sciences (M.K.K.), Toronto; Division of General Internal Medicine and Clinical Epidemiology (M.K.K.), University Health Network; and Institute for Clinical Evaluative Sciences (J.F.), Toronto, Ontario, Canada.

Although the RCSN is mentioned in the byline, a comprehensive list of organization members was not provided for this publication. Study funding: The Registry of the Canadian Stroke Network is funded by the Canadian Stroke Network, a National Centres of Excellence (NCE) program, and the Ontario Ministry of Health and Long-Term Care.

Disclosure: Author disclosures are provided at the end of the article. 
acute care institutions in 8 Canadian provinces. All participating institutions were tertiary care centers, with expertise in stroke care and with the capacity to administer thrombolysis.

Standard protocol approvals, registrations, and patient consents. In these first 2 phases of the RCSN, informed consent from the patient or surrogate was required for data collection, and was obtained in all patients included in the registry. This comprised approximately $40 \%$ of eligible patients. ${ }^{7}$ The common reasons for inability to obtain consent were 1) the patient died before consent could be obtained; 2) language barrier; 3) unavailability of a surrogate decision-maker. The RCSN is not registered on any international trial registries.

Data were collected prospectively by trained neurology research nurses through chart review and patient and provider interviews, using laptop computers and custom software. Information on pre-hospital, emergency department, and inhospital care was collected. Baseline stroke severity was captured using the Glasgow Coma Scale (for unconscious patients) and the Canadian Neurological Scale score (for conscious patients). Follow-up telephone interviews were performed at 6 months after the index stroke event to collect information on outcomes including functional status and quality of life. ${ }^{7}$ For the present analysis, the cohort was limited to patients with a final diagnosis of ischemic stroke. At each site, the protocol was reviewed and approved by the local research ethics board.

The primary outcomes were the Stroke Impact Scale-16 score $^{8,9}$ (SIS-16) and mortality at 6 months. Secondary outcomes included in-hospital mortality as well as length of hospitalization and discharge disposition. For the SIS-16, a good score was defined as a score $\geq 75$ points, which is equivalent to independent function or a modified Rankin Score (mRS) of 2 or less. ${ }^{8}$

We divided patients into those who received and those who did not receive thrombolytic therapy and compared the outcomes. We examined the data for a thrombolysis-by-sex interaction on the 2 primary outcomes using unconditional logistic regression analysis. We considered $p<0.10$ for the interaction term as indicative of statistically relevant evidence. Main effects variables were assessed at the customary $p<0.05$ level. Sex, age, stroke severity, and onset-to-treatment time were included in the model to adjust for known prognostic variables. Statistical analyses were performed using SAS 9.1 .

RESULTS The overall cohort included 2,113 patients, $43.5 \%$ of whom were female. Of these, 232 (11\%) were treated with thrombolysis. Compared to those who did not receive thrombolysis, patients who received thrombolysis were less likely to be on an antiplatelet therapy prior to admission, had more severe stroke, were more likely to be transported to the hospital by ambulance, and had faster onset-to-CT times. Clinical characteristics were similar in men and women, with the exception of slightly greater stroke severity, a lower prevalence of hyperlipidemia, and slightly lower mean hematocrit and blood glucose levels in women compared to men in both the thrombolyzed and nonthrombolyzed cohorts (table 1). In the thrombolysis cohort, women had slightly more severe stroke than men (median Canadian Neurological Score 5 for women vs 6 for men) (table 1).

Overall, mortality and functional status at 6 months poststroke were similar in men and women (table 2). Mortality in-hospital and at 6 months was slightly higher in the thrombolysis cohort compared to the nonthrombolyzed cohort but there was no difference in mortality between men and women within cohorts. In the group that did not receive thrombolysis, men were significantly more likely than women to achieve a good outcome (SIS-16>75) at 6 months poststroke ( $70 \%$ vs $58 \%, p<0.001)$. In contrast, men and women who received thrombolysis were equally likely to achieve a good outcome at 6 months. In a multiple logistic regression model, adjusting for age, stroke severity, and onset to emergency department times, we observed a significant interaction $(p=0.054$, Wald test) for the SIS-16 outcome and a nonsignificant interaction for 6-month mortality ( $p=0.722$, Wald test). These results were not different when we forced (because there were slight baseline differences between cohorts) hyperlipidemia, glucose, and hematocrit as covariables into the model. Main effects of age and stroke severity were significant predictors of both SIS-16 $>75$ and 6-month mortality. In an exploratory model, no sex-by-thrombolysis interaction effect was seen for in-hospital mortality.

DISCUSSION These data provide confirmatory evidence of a treatment-by-sex interaction in this cohort of patients routinely treated with thrombolysis. The direction of effect seen in this cohort study is identical to what has been previously reported: women fare poorly compared to men when not treated with thrombolysis, achieve outcomes similar to those seen in men when treated with thrombolysis, and thus have a larger absolute benefit from thrombolytic therapy. . $^{3,10}$

Why women not treated with thrombolysis have worse outcomes than men is not well understood, but it has been observed in other cohorts. ${ }^{11}$ What are the biologic differences that may account for this phenomenon? In our study, hyperlipidemia, hematocrit, and baseline serum glucose were numerically lower in the female cohort. Although these factors did not alter the multivariable regression results, preliminary preclinical evidence suggests that these factors may be relevant to thrombolysis. ${ }^{12,13}$ Other factors such as reduced thrombus burden, and therefore faster recanalization, have been suggested, but this has not been convincingly proven to date. ${ }^{14-18}$ One other study of the GAIN trial has suggested no gender effect, but this study is potentially biased by the exclusion of rapid responders to thrombolysis. ${ }^{19,20}$

The response to thrombolysis might depend on the mechanism of stroke. Recanalization is seen more often in the fibrin-rich cardioembolic strokes than the platelet-rich thromboses on preexisting atherosclerotic lesions, because of the high affinity of tPA for fibrin. ${ }^{21}$ Older women are more prone to cardio- 
Table 1 Patient characteristics ${ }^{a}$

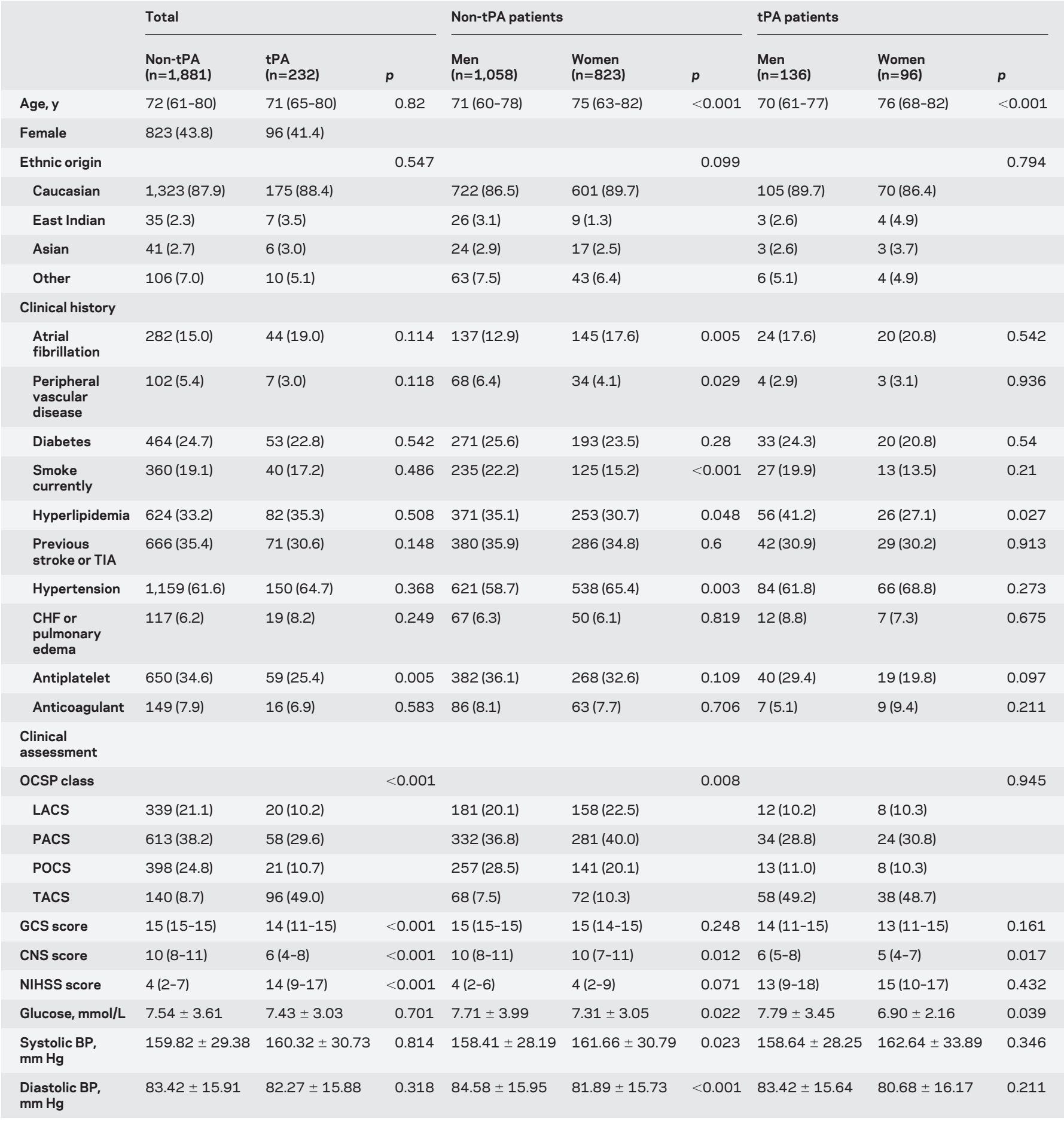

Abbreviations: $\mathrm{BP}=$ blood pressure; $\mathrm{CHF}=$ congestive heart failure; CNS = Canadian Neurological Scale; GCS = Glasgow Coma Scale; LACS = lacunar stroke; NIHSS = NIH Stroke Scale; OCSP = Oxfordshire Community Stroke Project; PACS = partial anterior circulation stroke; POCS = posterior circulation stroke; TACS $=$ total anterior circulation stroke; $\mathrm{tPA}=$ tissue plasminogen activator.

aValues are median (interquartile range), $\mathrm{n}(\%)$, or mean $\pm \mathrm{SD}$.

embolic strokes due to an increased prevalence of atrial fibrillation. ${ }^{22}$ However, in this study, atrial fibrillation was not seen more often in women than in men. Sex-related differences have been noticed in cardiovascular diseases as well consequent to the differences in the fibrinolytic status between men and women. ${ }^{23}$ There was a significant increase in symp- tomatic middle cerebral artery involvement in female patients as compared to male patients in the WASID study; this could explain the greater benefit of thrombolysis in female patients, and a worse prognosis if not treated. ${ }^{24}$

It is also possible that the impact is a social one. Whereas women may care for men, who tend to have 
Table 2 Mortality and functional status 6 months after stroke

\begin{tabular}{|c|c|c|c|c|c|c|c|c|c|}
\hline Variable & \multicolumn{3}{|l|}{ Total } & \multicolumn{3}{|c|}{ Non-tPA patients } & \multicolumn{3}{|c|}{ tPA patients } \\
\hline Follow-up sample size, $n$ & 1,740 & 201 & & 983 & 757 & & 120 & 81 & \\
\hline 6-month mortality, n (\%) & $72(5.0)$ & $14(8.1)$ & 0.081 & $36(4.4)$ & $36(5.6)$ & 0.3 & $8(7.8)$ & $6(8.5)$ & 0.885 \\
\hline SIS-16 > 75, n (\%) & $781(64.7)$ & $86(61.4)$ & 0.443 & $781(64.7)$ & $86(61.4)$ & $<0.001$ & 50 (61.7) & $36(61.0)$ & 0.932 \\
\hline
\end{tabular}

Abbreviations: SIS-16 = Stroke Impact Scale-16 score; tPA = tissue plasminogen activator.

stroke at a younger age, $31 \%$ of women are widowed compared to $7 \%$ of men at the time of stroke, and therefore they do not have a spouse who can act as a caregiver. ${ }^{25}$ Poststroke depression is more common in women than in men, ${ }^{26}$ and this hinders functional recovery.

The purpose of this analysis was to build further evidence to show a consistency of effect of the treatmentby-sex interaction. Consistency of effect is one of several factors that support a causal relationship. One limitation of our approach is that the methodology, a cohort design, is weaker than an examination of randomized clinical trial data. Despite the possibility of confounding by indication (patients who received thrombolysis were somehow different from those who did not, leading to differences in outcomes), the data are supportive of the proposed effect. Further work on understanding the relationship between sex and outcome after stroke is required.

\section{AUTHOR CONTRIBUTIONS}

The concept for the article was developed by Drs. Hill and Kapral. The primary draft was written by Drs. Hill, Sylaja, and Shobha. The statistical analysis was conducted by Dr. Fang. All authors reviewed and made critical revisions to the final manuscript.

\section{DISCLOSURE}

Dr. Shobha and Dr. Sylaja report no disclosures. Dr. Kapral holds a New Investigator Award from the CIHR, receives funding from the University Health Network Women's Health Program and the Canadian Stroke Network, and is a member of the GENESIS Team. Dr. Fang reports no disclosures. Dr. Hill has served/serves on the editorial boards of Neurology, Stroke, and the Canadian Journal of Neurological Sciences and as Editor of Stroke Rounds (Canada); has received honoraria from Boehringer Ingelheim, Roche, and sanofi aventis; receives research support from Roche, Bayer-Schering Pharma, the NIH/NINDS (U01 NS52220 [Co-I], U01 NS40406 [Co-PI]), the CIHR, the Canadian Stroke Network, the Alberta Heritage Foundation for Medical Research, and from the Heart \& Stroke Foundation of Alberta.

Received March 3, 2009. Accepted in final form December 7, 2009

\section{REFERENCES}

1. Kapral MK, Fang J, Hill MD, et al. Sex differences in stroke care and outcomes: results from the Registry of the Canadian Stroke Network. Stroke 2005;36:809-814.
2. Kapral MK, Wang H, Austin PC, et al. Sex differences in carotid endarterectomy outcomes: results from the Ontario Carotid Endarterectomy Registry. Stroke 2003;34:11201125.

3. Kent DM, Price LL, Ringleb P, Hill MD, Selker HP. Sexbased differences in response to recombinant tissue plasminogen activator in acute ischemic stroke: a pooled analysis of randomized clinical trials. Stroke 2005;36:62-65.

4. Hill MD, Kent DM, Hinchey JA, et al, on behalf of the PROACT-2 Investigators. Sex-based differences in the effect of intra-arterial treatment of stroke: analysis of the PROACT-2 Study. Stroke 2006;37:2322-2325.

5. Savitz SI, Schlaug G, Caplan L, Selim M. Arterial occlusive lesions recanalize more frequently in women than in men after intravenous tissue plasminogen activator administration for acute stroke. Stroke 2005;36:1447-1451.

6. Kapral MK, Silver FL, Richards JA, Lindsay P, Fang J, Shi HS. Registry of the Canadian Stroke Network. Progress Report 2001-2005. Toronto: Institute for Clinical Evaluative Sciences; 2005.

7. Tu JV, Willison DJ, Silver FL, et al. Impracticability of informed consent in the Registry of the Canadian Stroke Network. N Engl J Med 2004;350:1414-1421.

8. Duncan PW, Lai SM, Bode RK, Perera S, DeRosa J. Stroke Impact Scale-16: a brief assessment of physical function. Neurology 2003;60:291-296.

9. Edwards B, O'Connell B. Internal consistency and validity of the Stroke Impact Scale 2.0 (SIS 2.0) and SIS-16 in an Australian sample. Qual Life Res 2003;12:1127-1135.

10. Kent DM, Hill MD. Gender differences in tPA-related arterial recanalization. Stroke 2005;36:2529; author reply 2529-2530.

11. Saposnik G, Di Legge S, Webster F, Hachinski V. Predictors of major neurologic improvement after thrombolysis in acute stroke. Neurology 2005;65:1169-1174.

12. Duan J, Murohara T, Ikeda H, et al. Hypercholesterolemia inhibits angiogenesis in response to hindlimb ischemia: nitric oxide-dependent mechanism. Circulation 2000;102:III370-III376.

13. Poppe AY, Majumdar SR, Jeerakathil T, Ghali W, Buchan AM, Hill MD. Admission hyperglycemia predicts a worse outcome in stroke patients treated with intravenous thrombolysis. Diabetes Care Epub 2009.

14. Liebeskind D. Vessel size explains gender differences in response to intravenous thrombolysis for acute stroke. Stroke 2006;37:622.

15. Buck B, Kim D, Saver JL. The volume of middle cerebral artery thrombi: assessment by GRE MRI, gender differ- 
ences, and relation to response to fibrinolytic therapy. Stroke 2006;37:648.

16. Kain K, Catto AJ, Carter AM, et al. Decreased fibrinolytic potential in South Asian women with ischaemic cerebrovascular disease. Br J Haematol 2001;114:155-161.

17. Kain K, Carter AM, Bamford JM, Grant PJ, Catto AJ. Gender differences in coagulation and fibrinolysis in white subjects with acute ischemic stroke. J Thromb Haemost 2003; 1:390-392.

18. Shah SH, Liebeskind DS, Saver JL, et al. Influence of gender on outcomes after intra-arterial thrombolysis for acute ischemic stroke. Neurology 2006;66:1745-1746.

19. Elkind MS, Prabhakaran S, Pittman J, Koroshetz W, Jacoby M, Johnston KC. Sex as a predictor of outcomes in patients treated with thrombolysis for acute stroke. Neurology 2007;68:842-848.

20. Kent DM, Buchan AM, Hill MD. The gender effect in stroke thrombolysis: of CASES, controls, and treatment-effect modification. Neurology 2008;71:1080-1083.

21. Molina CA, Montaner J, Arenillas JF, Ribo M, Rubiera M, Alvarez-Sabin J. Differential pattern of tissue plas- minogen activator-induced proximal middle cerebral artery recanalization among stroke subtypes. Stroke 2004; 35:486-490.

22. Reeves MJ, Bushnell CD, Howard G, et al. Sex differences in stroke: epidemiology, clinical presentation, medical care, and outcomes. Lancet Neurol 2008;7:915-926.

23. Eriksson MA, Rask E, Johnson O, et al. Sex-related differences in the associations between hyperleptinemia, insulin resistance and dysfibrinolysis. Blood Coagul Fibrinolysis 2008;19:625-632.

24. Williams JE, Chimowitz MI, Cotsonis GA, Lynn MJ, Waddy SP. Gender differences in outcomes among patients with symptomatic intracranial arterial stenosis. Stroke 2007;38:2055-2062.

25. Foerch C, Misselwitz B, Humpich M, Steinmetz H, Neumann-Haefelin T, Sitzer M. Sex disparity in the access of elderly patients to acute stroke care. Stroke 2007;38: 2123-2126

26. Paradiso S, Robinson RG. Gender differences in poststroke depression. J Neuropsychiatry Clin Neurosci 1998; 10:41-47.

\section{CDC, AAN to Health Care Professionals: Monitor Patients for GBS}

The Centers for Disease Control and Prevention (CDC) and the American Academy of Neurology (AAN) collaborated to reach out to neurologists across the US to monitor and report any possible new cases of Guillain-Barré syndrome (GBS) following 2009 H1N1 flu vaccination.

Neurologists and health care professionals nationwide who diagnose patients with vaccineassociated GBS should use the CDC and FDA Vaccine Adverse Event Reporting System (VAERS) to report their observations.

In addition, neurologists and all health practitioners in the 10 Emerging Infections Program (EIP) states-California, Connecticut, Maryland, Minnesota, New Mexico, New York, Colorado, Oregon, Georgia, and Tennessee-are asked to report all new cases of GBS, regardless of vaccination status, to their state's surveillance officer.

The AAN hosted a series of webinars providing an in-depth look at H1N1 vaccination and how it may pose a risk for GBS and information about the vaccination monitoring campaign.

For additional information about the monitoring campaign, or to watch the webinars or download VAERS form and information on reporting to surveillance officers in your state, visit the AAN's GBS toolkit page, www.aan.com/view/gbstoolkit. 